\title{
Epidemiology, clinical features and diagnosis of Mycobacterium ulcerans in an Australian population
}

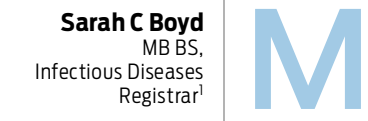

ycobacterium ulcerans causes a necrotising infection of skin and subcutaneous tissue and was first formally identified in 1948. After tuberculosis and leprosy, it is the third most common mycobacterial infection worldwide among immunocompetent individuals. ${ }^{1,2}$

While most $M$. ulcerans infections occur in sub-Saharan Africa, it is endemic in more than 30 countries. In Australia, it occurs focally in northern and sub-tropical areas and the temperate south-eastern region. In Victoria it is known as Bairnsdale ulcer, and elsewhere as Daintree ulcer or Buruli ulcer. Cases in Victoria's Bellarine Peninsula were first reported in 1998, and this is now recognised as an endemic area with the highest burden of disease in Australia. ${ }^{3}$

The mode of transmission and risk factors for $M$. ulcerans infection remain unclear. Infection may be acquired from an environmental source, possibly from exposure to contaminated soil or vegetation or by aerosol inhalation. Endemic areas are typically located near large bodies of water, and recent evidence has implicated aquatic organisms and mosquitoes in disease transmission, and terrestrial mammals as potential reservoir species. ${ }^{3-8}$

There are two clinical stages of $M$. MJA 2012; 196: 341-344 doi: 10.5694/mjal2.10087 ulcerans infection - preulcerative and ulcerative disease. ${ }^{9-11}$ Preulcerative disease forms include nodules, plaques and oedematous lesions that develop into painless ulcers with characteristic undermined edges. Systemic features are typically absent. $^{12,13}$ All age groups may be affected by disease; however, in countries with the highest disease burden, the majority of cases affect young children. ${ }^{14,15}$

Detailed information on the epidemiology and clinical features of $M$. ulcerans in south-eastern Australia is lacking, and it is possible that the temperate climate and preponderance of older residents may result in differences

Abstract

Objective: To describe the epidemiology, clinical features and diagnosis of Mycobacterium ulcerans infection occurring on the Bellarine Peninsula in Victoria.

Design, setting and patients: Analysis of prospectively collected data on all patients with confirmed $M$. ulcerans infection reported from the Bellarine Peninsula and managed at Barwon Health between 1 January 1998 and 2 September 2011.

Main outcome measures: Proportion of cases by age group and sex; duration of symptoms; number, type and site of lesions; proportions diagnosed by polymerase chain reaction (PCR) testing and mycobacterial cultures.

Results: We identified 180 sequential cases of $M$. ulcerans infection: 42 cases in 1998-2004 and 138 in 2005-2011. Median patient age was 61 years (range, 1-94 years), and $49 \%$ of patients were male. Duration of symptoms before diagnosis varied from 2 to 270 days (median, 42 days). At presentation, 95\% of patients had single lesions. Lower limbs were the most common site of lesions (61\%), followed by upper limbs (34\%); 40\% occurred over a joint. Most lesions presented as ulcers ( $87 \%$ ). Compared with the rest of the study population, patients aged $>60$ years were more likely to have multiple lesions (odds ratio [OR], 8.1; $95 \% \mathrm{Cl}, 1.0-176.2 ; P=0.04)$, and patients aged $<15$ years were less likely to have lesions over a joint (OR, 0.2; 95\% Cl, 0.0-1.0; $P=0.02)$. Upper limb lesions were more common (OR, 2.2; 95\% Cl, 1.1-4.3; $P=0.02$ ) and lower limb lesions less common (OR, $0.5 ; 95 \% \mathrm{Cl}, 0.5-0.9 ; P=0.01)$ in male than female patients. Lesion swabs were PCR-positive in $99 \%$ of those tested, and positive on mycobacterial culture in 19\%. Lesion biopsy samples were PCR-positive in 95\% and mycobacterial culture-positive in 47\%.

Conclusions: M. ulcerans infections are increasing in the Bellarine Peninsula. They usually present as single ulcerative lesions on limbs, commonly over joints. Age and sex influence clinical presentation, and PCR of lesions has high diagnostic sensitivity.

with tropical endemic regions. Our aim was to describe the epidemiology, clinical features and diagnosis of M. ulcerans infection occurring on the Bellarine Peninsula, and to compare the epidemiology and clinical features with descriptions from tropical endemic regions in Africa.

\section{Methods}

Data were prospectively collected on all confirmed cases of $M$. ulcerans infection managed by the infectious diseases service at Barwon Health between 1 January 1998 and 2 September 2011. A case of M. ulcerans infection was defined by the presence of a lesion clinically suggestive of $M$. ulcerans infection, plus any one of: - Culture of M. ulcerans from a lesion swab or biopsy sample (performed using Lowenstein-Jensen media and incubated for 12 weeks)
- Positive polymerase chain reaction (PCR) test result on lesion swab or biopsy sample

- Histopathological lesion sample showing a necrotic granulomatous ulcer with the presence of acid-fast bacilli (AFB).

Data variables collected included patient age, sex and residential address; date and method of M. ulcerans diagnosis; site, type and number of lesions; duration of symptoms; and comorbid conditions. Geographic location of a case was defined as the most likely place of acquisition, determined by residential address if living in a known endemic area or place visited if living outside the endemic area.

Ethics approval was obtained from the Barwon Health Research and Ethics Advisory Committee.

\section{Statistical analysis}

Data analysis was performed using Epi Info 6 (Centers for Disease Control 
1 Number, clinical site and type of Mycobacterium ulcerans lesions, by patient age and sex

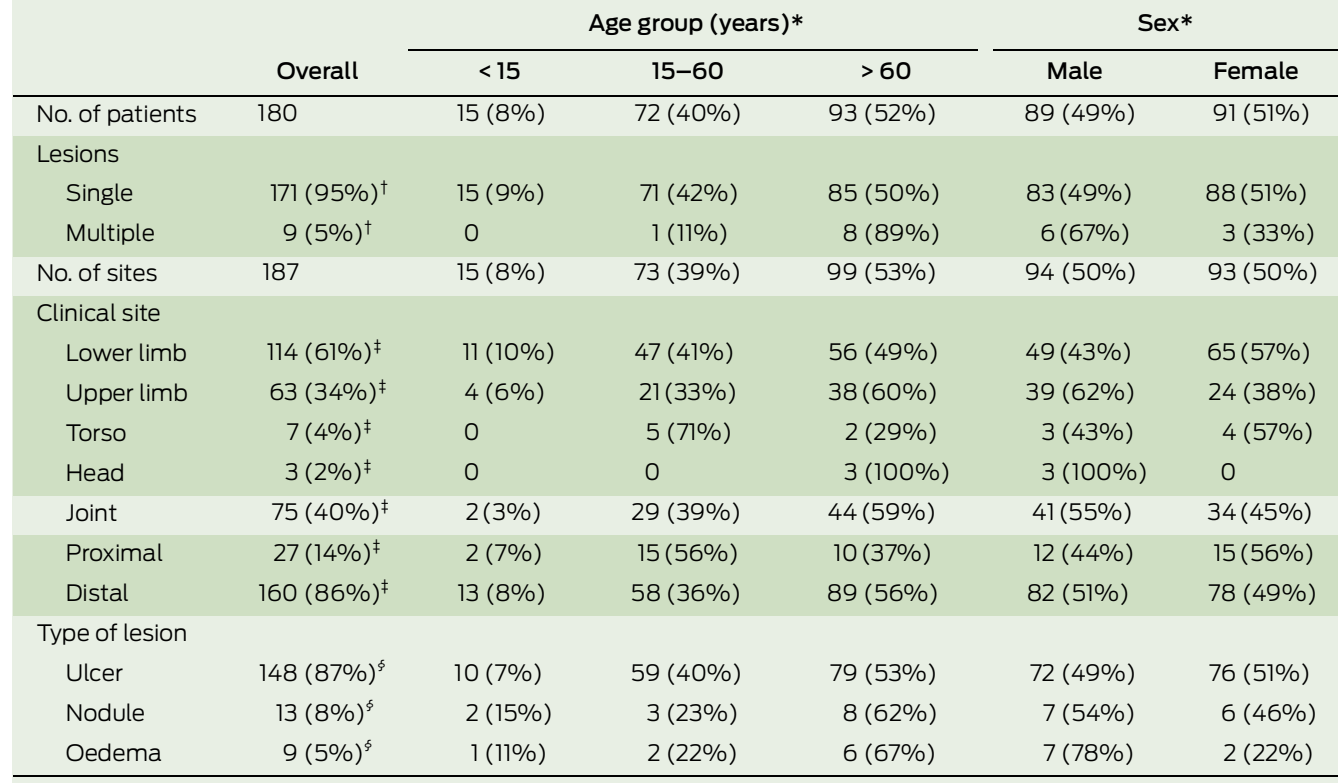

* Denominators for percentages in age group and sex columns are the overall totals for each row. $\dagger$ Denominator is total number of patients. $\ddagger$ Denominator is total number of sites. $\oint$ Denominator is total number of lesions where type was recorded $(n=170)$.

2 Location of 180 Mycobacterium ulcerans cases, Bellarine Peninsula, 1998-2011

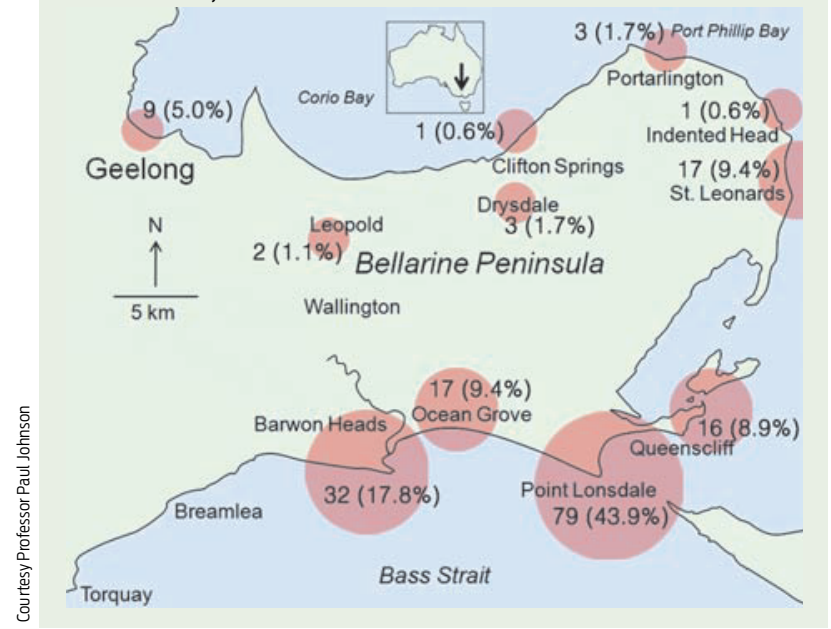

and Prevention, Atlanta, Ga, USA). Statistical comparisons of proportions were determined using the two-tailed Fisher exact test, and median values were compared using the MannWhitney test.

\section{Results}

We identified 180 sequential cases of M. ulcerans infection for analysis.

\section{Epidemiology}

Cases and lesions stratified by age and by sex are shown in Box 1. Median patient age at the time of diagnosis was 61 years (range, 1-94 years), and was similar for men (60 years) and women (63 years).

Most cases occurred in focal coastal towns, particularly Point Lonsdale (79; 44\%) and Barwon Heads (32; $18 \%$ ), with inland areas poorly represented (Box 2).

M. ulcerans infection was diagnosed year-round, with a peak incidence in winter months: 75 patients (42\%) presented in winter, 41 (23\%) in spring, 39 $(22 \%)$ in autumn, and $25(14 \%)$ in summer. Although the annual case number varied, case numbers increased overall, from 42 in 1998-2004 (average, 6 per year) to 138 in 2005-2011 (average, 20 per year) (Box 3 ).

\section{Clinical features}

The duration of symptoms before diagnosis varied from 2 to 270 days (median, 42 days), with no statistically significant difference between 19982004 and 2005-2011 (median [interquartile range], 60 days [28-75 days] v 40 days [24-70 days]; $P=0.21$ ).

Most patients had single lesions at presentation (Box 1), with multiple lesions observed in only nine patients: five had two lesions, three had three lesions, and one had 13 lesions.

In the 170 cases where type of lesion was recorded (Box 1), ulcerative lesions (Box 4) were most common, with fewer patients presenting with non-ulcerative disease, including nodular and oedematous (Box 5) lesions. Extension to deeper structures occurred in two patients $(1 \%)$ : one each to bone and bursa. The median duration of symptoms for ulcerative and non-ulcerative lesions was similar ( $54 \mathrm{v} 48$ days; $P=0.33$ ).

Lower limbs were the most common site of lesions (Box 1), with a predominance on the lower leg (57/ $187,30 \%)$ and ankle (27/187, 14\%), followed by upper limbs, mostly at the elbow (27/187, 14\%). Forty per cent of lesions were located over a joint.

Compared with the rest of the study population, lesions located over a joint were less common among patients aged $<15$ years (odds ratio [OR], 0.2; 95\% CI, 0.0-1.0; $P=0.02)$, proximal lesions were more common in patients aged 15-60 years (OR, 2.2; 95\% CI, 0.9-5.4; $P=0.06$ ), and patients aged $>60$ years were more likely to have multiple lesions (OR, 8.1; 95\% CI, 1.0 176.2; $P=0.04)($ Box 1$)$.

Upper limb lesions were more common (OR, 2.2; 95\% CI, 1.1-4.3; $P=0.02)$ and lower limb lesions less common (OR, 0.5; 95\% CI, 0.5-0.9; $P=0.01)$ in male than female patients.

Medical comorbidities included diabetes in 13 of 143 patients (9\%) and known malignancy in eight of 140 (6\%). There were no known HIVpositive patients.

\section{Diagnosis}

PCR test results were positive for 127 of 128 swab specimens (99\%) and 41 of 43 tissue biopsy samples (95\%). The single negative PCR swab case was diagnosed by a positive PCR result on a subsequent biopsy sample. One of the negative PCR biopsy cases was diagnosed by a positive PCR swab result, and the other by histopathological examination. Smears for AFB were positive in 28 of 49 swab specimens $(57 \%)$ and 49 of 63 biopsy specimens $(78 \%)$. Culture for $M$. ulcerans was positive in nine of 47 swab specimens (19\%) and 17 of 36 biopsy specimens (47\%).

\section{Discussion}

Our study shows that case numbers of M. ulcerans infection have been increasing since it emerged in St Leonards on the Bellarine Peninsula in 
1998. The disease has spread to other regional coastal towns, with the highest number of cases in Point Lonsdale and Barwon Heads. Inland areas have been relatively spared, and coastal towns further west, such as Breamlea and Torquay, remain unaffected. The focal coastal distribution suggests potential geographically confined environmental factors important for both survival of the organism and transmission of disease, which are yet to be determined and are important areas for future research. ${ }^{4}$

In our study population, all age groups were affected and the malefemale balance of disease was equal, similar to African studies. The majority of cases occurred in older people, which contrasts with the age-related disease distribution in Africa. 2,16,17 Children aged $<15$ years carry the highest burden of disease in Africa (up to $62 \%$ of cases $^{9}$ ), although they also comprise the majority of the local population. ${ }^{12}$ The Bellarine Peninsula's permanent population includes a large proportion of older people. More recent African data suggest a bimodal pattern of disease, with incidence peaking in both the 10-15years and 75-79-years age groups., ${ }^{2,16}$ Advanced age has also been implicated as an independent risk factor for disease, ${ }^{12,16}$ possibly related to agerelated immunosuppression. We do not have accurate population data for the Bellarine Peninsula and are unable to determine the exact age-related incidence of disease in this area, but previous research has reported mean annual incidences of 4.04 and 0.87 per 1000 people for Point Lonsdale and Barwon Heads, respectively. ${ }^{3}$

Most symptoms began, and cases were diagnosed, between autumn and spring, with a peak in winter. A Victorian study from Phillip Island reported a larger proportion of cases during cooler months (April to September) than warmer months (October to May), ${ }^{4}$ and our data are in keeping with this observation. A potential reason for a predominance of cases in these months is increased exposure to soil and insect vectors during warmer summer months, when less clothing is worn, followed by a prolonged incubation period. Incubation periods of up to 7 months have previously been reported. ${ }^{3,18}$
Alternatively, the seasonal nature of presentations may be related to higher rainfall during cooler months bringing soil-borne $M$. ulcerans organisms to the surface, combined with a short incubation period.

The relatively early presentation and diagnosis in our study population may explain the low number of patients with deep structure involvement. Our study's osteomyelitis rate of $1 \%$ contrasts with rates of up to $13 \%$ in African communities. ${ }^{11}$ Reasons for delayed disease presentations in Africa are likely multifactorial, including the clinical form of disease (poor recognition of early, non-ulcerative lesions, and the painless nature and slow progression of ulcerative lesions), cultural beliefs and social stigma, and difficulty in accessing appropriate health care. ${ }^{2,19}$ Patients in Australia also poorly recognise the non-ulcerative form of disease and delay seeking medical care until the characteristic ulcerative lesions develop. ${ }^{20}$ Increased community and health care provider awareness of $M$. ulcerans is associated with earlier presentation and diagnosis. ${ }^{11,20}$ The importance of increased awareness of symptoms has been emphasised in reducing delays to presentation, patient morbidity, disease severity and treatment costs. ${ }^{6,21,22}$

Our data confirm that M. ulcerans infection occurs mostly in distal limbs, with lesion distribution in our Australian population similar to that in patients in Africa, where $66 \%$ of one case series of 5772 lesions were located on lower limbs. ${ }^{23}$ However, there are notable differences between Australian and African communities in the number and location of lesions in age groups and by sex. Unlike our population, upper limb involvement was found to be less common in patients over 15 years of age in Africa. ${ }^{16}$ In our study, upper limb lesions were more common in male patients, while African data report upper limb involvement as more common in female patients. ${ }^{16,23}$ Proximal lesions were noted to be more frequent in children and young adults (aged $<20$ years) in Benin ${ }^{16}$ and Ghana, ${ }^{23}$ but we found the highest frequency of proximal lesions in the 15-60-years age group. Further, we found an increased frequency of
3 Year of diagnosis of Mycobacterium ulcerans cases, Bellarine Peninsula, 1998-2011
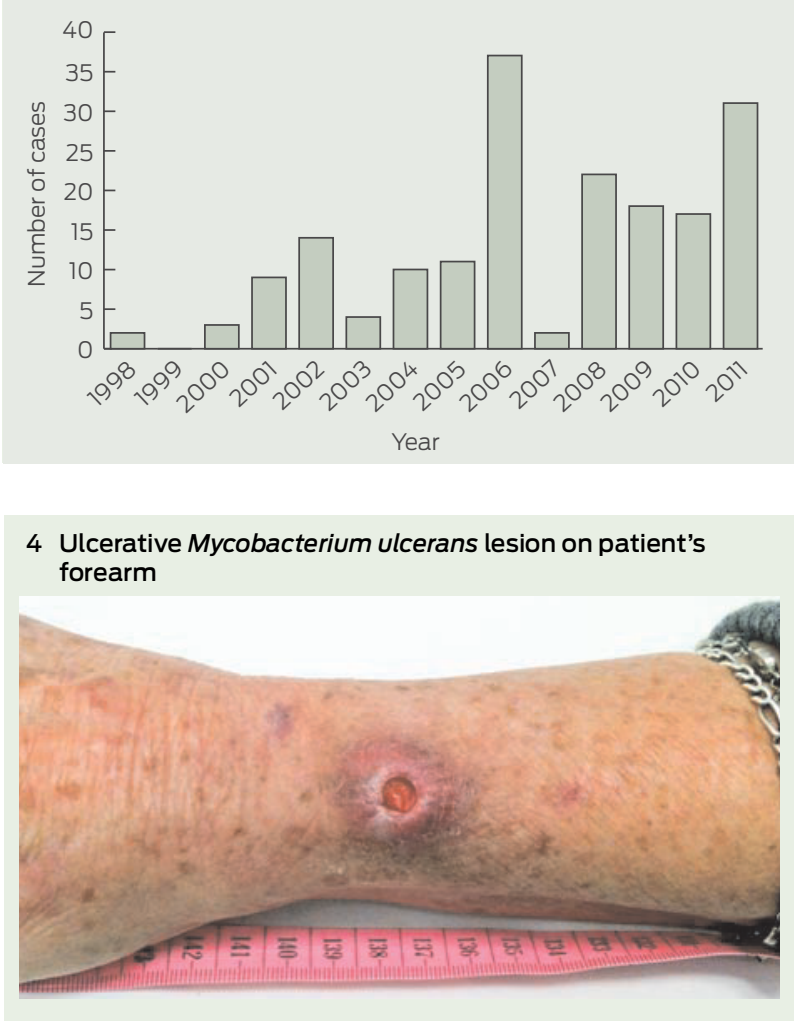

5 Oedematous Mycobacterium ulcerans lesion surrounding central ulcerative lesion on patient's elbow

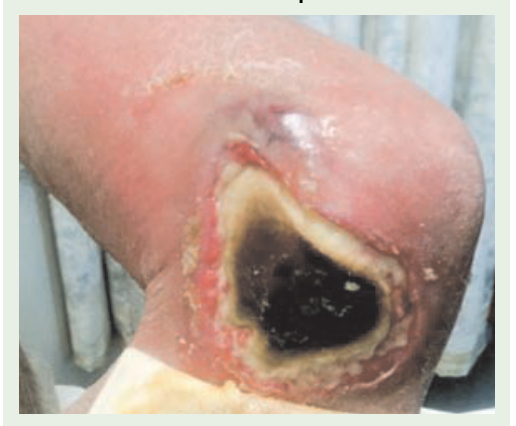

multiple lesions in patients aged $>60$ years, while patients aged $<30$ years in Benin had the highest prevalence of multiple lesions (10.7\%). ${ }^{16}$ Multiple lesions were reported to affect $32 \%$ of 121 patients in Ghana, and, although no age comparison was provided, $62 \%$ of the patient population were aged $<15$ years. $^{9}$

These differences between our patient population and African populations could be due to age-related or genetic differences. Previous research has identified decreasing environmental exposure (eg, wearing long pants) as being protective against 
infection, ${ }^{7,9}$ and exposure and lifestyle differences between Australia and Africa may result in variable disease manifestations. Furthermore, there may be innate differences in the clinical behaviour of Australian strains of M. ulcerans. Mycolactone toxins, responsible for the pathogenicity of M. ulcerans, are more diverse and produced in greater quantities in African strains of $M$. ulcerans than in Australian strains, possibly contributing to their higher virulence. ${ }^{24}$

Lesions were located over a joint in $40 \%$ of our patient population, possibly due to minor skin trauma and increased environmental exposure providing a point of entry for infection at these locations. To date, there are no published data from Africa regarding the frequency of lesions over joints.

We found PCR to be highly sensitive for diagnosis on both swab and biopsy samples of lesions. PCR has previously been reported as approaching $100 \%$ specificity and $96 \%$ sensitivity for lesion swabs, ${ }^{25,26}$ and our data support PCR as the test of choice for diagnosis. Mycobacterial cultures were less sensitive for diagnosis, although positive cultures were more commonly obtained from tissue biopsy than swab specimens. However, cultures remain important for epidemiological typing and monitoring for antibiotic resistance.

The current management practice at Barwon Health for M. ulcerans infections is to initially offer all patients combination oral antibiotics containing rifampicin plus either a fluoroquinolone (moxifloxacin or ciprofloxacin) or clarithromycin. ${ }^{27}$ The choice of fluoroquinolone or clarithromycin is based on factors such as age, comorbidities, predicted drug tolerance, potential for drug interactions, and pregnancy. If no deep structures are involved and antibiotics are tolerated, they are given for 8 weeks. Debridement surgery is offered if it would be beneficial for wound healing (eg, large tissue necrosis); to prevent significant scarring or deformity; if antibiotics are contraindicated, refused or not tolerated after less than 4 weeks' total treatment; or at the request of patients to hasten wound healing. Ideally, antibiotics are administered for at least 4 weeks before surgery. Wound closure is achieved by direct suture, free skin graft or flap, or wounds are allowed to heal secondarily. Patients are monitored for paradoxical reactions, and suspicious lesions are ideally confirmed by histopathological examination. ${ }^{28}$ Treatment of mild paradoxical reactions is with observation alone, but if the reaction is severe and threatening significant tissue destruction or the integrity of skin grafts, a short course of prednisolone is given (0.5-1 mg/kg daily, weaned over $4-8$ weeks).

In conclusion, M. ulcerans infections on the Bellarine Peninsula are increasing in frequency but remain localised to focal coastal communities. In this population, disease usually presents as a single ulcerative lesion on distal limbs, commonly over joints, and extension to deeper structures is uncommon. Clinical presentation varies by age and sex, with multiple lesions more common in older people, lesions over a joint less common in children, and higher proportions of lesions on upper limbs in male patients and lower limbs in female patients. PCR testing of lesion swabs or biopsy specimens has high sensitivity for diagnosis.

Acknowledgements: We thank Mr Yu Zhang for his assistance with statistical analysis, Professor Paul Johnson for providing a map of the Bellarine Peninsula and Mr Dallas Wilson for assisting with preparation of figures.

Competing interests: No relevant disclosures.

Received 13 Jan 2012, accepted 26 Feb 2012.

1 Pszolla N, Sarkar MR, Strecker W, et al. Burul ulcer: a systemic disease. Clin Infect Dis 2003; 37: e78-e82.

2 Sizaire V, Nackers F, Comte E, Portaels F. Mycobacterium ulcerans infection: control diagnosis, and treatment. Lancet Infect Dis 2006 6:288-296.

3 Lavender CJ, Fyfe JA, Azuolas J, et al. Risk of Buruli ulcer and detection of Mycobacterium ulcerans in mosquitoes in southeastern Australia. PLoS Negl Trop Dis 2011; 5: el305.

4 Veitch MGK, Johnson PDR, Flood PE, et al. A large localized outbreak of Mycobacterium ulcerans infection on a temperate southern Australian island. Epidemiol Infect 1997; 119: 313-318.

5 Marsollier L, Deniaux E, Brodin P, et al. Protection against Mycobacterium ulcerans lesion development by exposure to aquatic insect saliva. PLoS Med 2007; 4: e64.

6 Johnson PDR, Stinear T, Small PLC, et al. Buruli ulcer (M. ulcerans infection): new insights, new hope for disease control. PLoS Med 2005; 2: el08.

7 Quek TYJ, Athan E, Henry MJ, et al. Risk factors for Mycobacterium ulcerans infection, southeastern Australia. Emerg Infect Dis 2007; 13: 1661-1666.

8 Fyfe JAM, Lavender CJ, Handasyde KA, et al. A major role for mammals in the ecology of Mycobacterium ulcerans. PLoS Negl Trop Dis 2010; 4: e791.
9 Raghunathan PL, Whitney EA, Asamoa K, et al. Risk factors for Buruli ulcer disease (Mycobacterium ulcerans infection): results from a case-control study in Ghana. Clin Infect Dis 2005; 40: 1445-1453.

10 van der Werf TS, van der Graaf WTA, Tappero JW, Asiedu K. Mycobacterium ulcerans infection. Lancet 1999; 354: 1013-1018.

11 Debacker M, Aguiar J, Steunou C, et al. Mycobacterium ulcerans disease (Buruli ulcer) in rural hospital, Southern Benin, 1997-2001. Emerg Infect Dis 2004; 10: 1391-1398.

12 Debacker M, Portaels F, Aguiar J, et al. Risk factors for Buruli ulcer, Benin. Emerg Infect Dis 2006; 12: 1325-1331.

13 Guarner J, Bartlett J, Whitney EAS, et al. Histopathologic features of Mycobacterium ulcerans infection. Emerg Infect Dis 2003; 9: 651-656.

14 Wansbrough-Jones M, Phillips R. Buruli ulcer: emerging from obscurity. Lancet 2006; 367: 1849-1858.

15 World Health Organization. Buruli ulcer: progress report 2004-2008. Wkly Epidemiol Rec 2008; 83: 145-156.

16 Debacker M, Aguiar J, Steunou C, et al. Mycobacterium ulcerans disease: role of age and gender in incidence and morbidity. Trop Med Int Health 2004; 9: 1297-1304.

17 Sopoh GE, Johnson RC, Chauty A, et al. Burul ulcer surveillance, Benin 2003-2005. Emerg Infect Dis 2007; 13: 1374-1376.

18 Lavender CJ, Senanayake SN, Fyfe JAM, et al. First case of Mycobacterium ulcerans disease (Bairnsdale or Buruli ulcer) acquired in New South Wales. Med J Aust 2007; 186: 62-63.

19 Stienstra Y, van der Graaf WTA, Asamoa K, van der Werf TS. Beliefs and attitudes towards Buruli ulcer in Ghana. Am J Trop Med Hyg 2002; 67: 207-213.

20 Quek TYJ, Henry MJ, Pasco JA, et al. Mycobacterium ulcerans infection: factors influencing diagnostic delay. Med J Aust 2007; 187: 561-563.

21 Drummond C, Butler JR. Mycobacterium ulcerans treatment costs, Australia. Emerg Infect Dis 2004; 10: 1038-1043.

22 Pak J, O'Brien DP, Quek T, Athan E. Treatment costs of Mycobacterium ulcerans in the antibiotic era. Int Health Epub 2012 Feb 16. doi: 10.1016/ j.inhe.2011.12.005.

23 Amofah F, Bonsu F, Tetteh C, et al. Buruli ulcer in Ghana: results of a national case search. Emerg Infect Dis 2002; 8: 167-170.

24 Mve-Obiang A, Lee RE, Portaels F, Small PL. Heterogeneity of mycolactones produced by clinical isolates of Mycobacterium ulcerans: implications for virulence. Infect Immun 2003; 71 : 774-783.

25 Johnson PDR, Hayman JA, Quek TY, et al; Mycobacterium ulcerans Study Team. Consensus recommendations for the diagnosis, treatment and control of Mycobacterium ulcerans infection (Bairnsdale or Buruli ulcer) in Victoria, Australia. Med J Aust 2007; 186: 64-68.

26 Phillips R, Horsfield C, Kuijper S, et al. Sensitivity of PCR targeting the IS2404 insertion sequence of Mycobacterium ulcerans in an assay using punch biopsy specimens for diagnosis of Buruli ulcer. J Clin Microbiol 2005; 43: 3650-3656.

27 O'Brien DP, McDonald A, Callan P, et al. Successful outcomes with oral fluoroquinolones combined with rifampicin in the treatment of Mycobacterium ulcerans: an observational cohort study. PLoS Negl Trop Dis 2012; 6: el473.

28 O'Brien DP, Robson ME, Callan PP, McDonald AH. "Paradoxical" immune-mediated reactions to Mycobacterium ulcerans during antibiotic treatment: a result of treatment success, not failure. Med J Aust 2009; 191: 564-566. 